\title{
Transvaginal Ultrasonographic Findings of Infertile Females in Population of Lahore
}

\author{
Ayesha Ahmed * Anjum Tazeen Sabeen Ahmad Mehreen Fatima S Muhammad Yousaf Farooq \\ Hafsa Zahid Ali Zahid Mahnoor Rafique Ayesha Irshad \\ University Institute of Radiological Sciences and Medical Imaging Technology, Faculty of Allied Health \\ Sciences, University of Lahore, Lahore, Pakistan.
}

\begin{abstract}
:
Failure of conception in women after frequent unprotected intercourse for one year is known as infertility. Ultrasound examination can detect certain pathologies that may result in primary or secondary infertility.

Objective:

To describe the various transvaginal ultrasonographic findings of infertile females in population of Lahore.

Methods:

The study was started after the consent of ethical committee of the University of Lahore. All the patients were registered in this study after signing the informed consent form. Toshiba Xario with transvaginal transducer frequency ranging from 9-12 $\mathrm{MHz}$ was used. Pathologies were evaluated through transvaginal scanning and sonographic data was kept in the ultrasound machine. A consecutive sampling technique was used and data was further evaluated with the help of Statistical Package for the Social Sciences version 24.
\end{abstract}

Results:

Among 138 females, PCOS was seen in $40.6 \%$ of the subjects. The second most common pathology was ovarian cyst which was seen in $10.9 \%$ of subjects. And the incidence of multiple fibroids was $5.1 \%$ as third most common pathology in both primary and secondary infertility cases. Most ovarian pathologies were found to be bilateral. $12.3 \%$ subjects had no findings on ultrasound.

Conclusion: PCOS was the utmost common pathology connected with primary and secondary infertility. The second and third most common pathologies were ovarian cyst and multiple fibroids respectively.

Keywords: female infertility, PCOS, PID

DOI: $10.7176 / \mathrm{JBAH} / 10-12-05$

Publication date:June $30^{\text {th }} 2020$

\section{Introduction}

Failure of conception in women after frequent unprotected intercourse for one year is known as infertility ${ }^{1}$. It is a historic problem impacting women's health and lifestyle causing social and psychological stress leading to agony and insecurity in their life ${ }^{2}$. The prevalence of female infertility among all infertile couples is $37 \%$ globally $^{3}$. However, in Pakistan the prevalence of infertility is about $22 \%$ with $4 \%$ primary and $18 \%$ secondary infertility ${ }^{4}$. During the evaluation of infertile female the commencement of examination and background must include history of menstrual cycle, frequency and timing of intercourse, use of contraceptive medicines or devices, infection of pelvic cavity, previous pregnancies and their results, use of other medications, occupational exposures, abuse of substances, alcohol consumption, tobacco consumption, and any surgery of pelvic organs ${ }^{5}$. Fallopian tube, endometrial cavity and ovaries involvement in infertility are $92-100 \%, 50 \%, 10-30 \%$ respectively, whereas Genital Tuberculosis is rare ${ }^{6}$. GTB is a chronic infection which leads to infertility by producing deleterious effects on fallopian tube causing irremediable damage, it may be asymptomatic or present with undetermined symptoms ${ }^{7}$. Various risk factor associated with female infertility are advanced age ( $>35$ years), elevated body mass index, age of onset of sexual activity, prior pelvic surgeries, stress, smoking, chemical and radiation exposure, insecticides, PID, contraceptive use, alcohol intake and drug abuse ${ }^{8}$. Infectious agents such as bacteria, viruses and other parasites affect normal human physiology and thus interfere with the normal process of reproduction ${ }^{9}$. Infertility is also caused by endocrine disorders such as different types of thyroid, ovarian, hypothalamic, pituitary and adrenal abnormalities ${ }^{10}$. Transvaginal sonography is a first line standard procedure for the determination of cause of infertility, whereas Pelvic ultrasonography is helpful in the diagnosis of PCOS, PID, fibroids, endometriosis, adenomyosis and abnormalities like ovarian cysts etc. Transvaginal sonography is cheap, free of radiation, completely safe, non-invasive, redoable, easy to perform and highly effective all around the world ${ }^{11}$. In female infertility, laparoscopy, hysteroscopy, hysterosalpingiography and transvaginal ultrasound are the most satisfying procedures for assessment of pelvic abnormalities ${ }^{12}$. Transvaginal sonography is better than transabdominal sonography in diagnosing subtle irregularities with better imaging quality and it is usually used simultaneously with transabdominal ultrasound but not as a replacement ${ }^{13}$. Transabdominal ultrasonography cannot detect all pathologies causing hindrance in conception but transvaginal ultrasonography is more efficient in detecting causes of female infertility so problem can be solved. The purpose of this study is to evaluate all pathologies that can be diagnosed by transvaginal ultrasonography in the work up of female infertility to describe the spectrum of 
diagnostic findings. In this study we will describe the frequency of pelvic pathologies that can be detected on TVS. In a study conducted by Theresa Widrich, the significance of ultrasonography and predominantly of transvaginal sonography in the setting of diagnosis and treatment of infertility is explained. Transabdominal ultrasound gives a wide view of the pelvic cavity however transvaginal ultrasonography provides more convenience and assistance in the field of gynecology. TVUS is considered as the obligatory gadget in the work-up of gynecologic infertility such as the evaluation of the female pelvic cavity, supervision of ovulation and oocyte collection for in-vitro fertilization procedure. The further extension in the field of ultrasonography is its use with contrast media which circumvent the application of hysteroscopy and laparoscopy in specific cases. Advances in ultrasound such as Color Doppler, Color Power Angio (CPA) and three-dimensional USG can play an important role in the assessment of infertile patients ${ }^{14}$.In a study, Fraser MA. et al, illustrated the rolr of transvaginal ultrasonography in the detection of endometriosis. TVUS is considered as the first-line imaging modality for the diagnosis of endometriosis due to its enhanced identification rate and administration of preoperative evaluation regarding the intensity of the disease. High standard of precision was shown in the diagnosis of endometriosis when a better quality of TVUS was performed by a specialist. In evaluating the diagnostic reliability of TVUS in deep endometriosis, it has a sensitivity of $77.5 \%$ as compared to the standard pelvic ultrasonography which is not conclusive to exclude non ovarian form of endometriosis. It also contributes in the illustration of comprehensive categorization of severity and intensity of the disease that may aid in the surgical preparation and guiding the patient $^{15}$. In a study conducted by M.Brassard. et al, the role of PCOS causing infertility in women is elaborated. PCOS is known to be one of the most common causes of infertility in women although it can be treated medically. It is typically found in young females. Almost $70 \%$ cases of PCOS cause a type of infertility in which ovulation is hindered. A well known efficacious method being used for the treatment of PCOS is the control of blood sugar level along with weight loss by exercise or diet. Weight loss is very effectual regarding the improvement in conception and also decreases the rate of unfavorable consequences of pregnancy corresponding to obesity ${ }^{16}$.

\section{Methods}

A Descriptive Cross-Sectional study was conducted at Gilani Ultrasound Centre and University Ultrasound Clinic Lahore. During 3 months of research period 138 females with the complain of primary and secondary infertility were willingly recruited. Examination was performed by using American Institute of Ultrasound in Medicine (AIUM) transvaginal ultrasound guidelines. Ultrasound machine Toshiba Xario with Transvaginal robe of frequency 9-12 MHz was used.

\section{Results}

A total of 138 participants including primary and secondary infertile individuals were recruited in this study. Out of them $72(52.2 \%)$ presented with the history of primary infertility and the remaining $66(47.8 \%)$ with secondary infertility, Table 1 .

\begin{tabular}{|c|c|c|}
\hline Primary/secondary infertility & Frequency & Percent \\
\hline P & 72 & $52.2 \%$ \\
\hline S & 66 & $47.8 \%$ \\
\hline Total & 138 & $\mathbf{1 0 0 . 0 \%}$ \\
\hline
\end{tabular}

Table 1. Frequency dispersal of primary/secondary infertility amid 138 women

Out of 138 individuals, pathologies were diagnosed in $121(87.7 \%)$ patients, while in $17(12.3 \%)$ patients no pathology was found, Table 2

\begin{tabular}{l|c|c|}
\hline Presence or absence of pathology & Frequency & Percent \\
\hline Absent & 17 & $12.3 \%$ \\
Present & 121 & $87.7 \%$ \\
Total & 138 & $\mathbf{1 0 0 . 0 \%}$ \\
\hline
\end{tabular}

Table 2. Frequency distribution for presence or absence of pathology.

The age group in years of study range from 10 minimum to 41 maximum with mean age at that time being 28.1449 years and the standard deviation being 5.42440 . The gravidity among the females of study group ranges from 0 minimum and 5 maximum with mean gravidity being 0.8333 and standard deviation being 1.11776 . The parity of the females ranges from 0 minimum and 3 maximum with mean parity being 0.4928 and standard deviation being 0.81273 . The number of abortions/miscarriage ranges from 0 minimum and 4 maximum with mean abortions/miscarriage being 0.3478 and standard deviation being 0.73148 , Table 3 . 


\begin{tabular}{|c|c|c|c|c|c|c|}
\hline Descriptive Statistics & $\mathbf{N}$ & Range & Minimum & Maximum & Mean & Std. Deviation \\
\hline Age & 138 & 31.00 & 10.00 & 41.00 & 28.1449 & 5.42440 \\
\hline Gravidity & 138 & 5.00 & 0.00 & 5.00 & 0.8333 & 1.11776 \\
\hline Parity & 138 & 3.00 & 0.00 & 3.00 & 0.4928 & 0.81273 \\
\hline Abortion/Miscarriage & 138 & 4.00 & 0.00 & 4.00 & 0.3478 & 0.73148 \\
\hline
\end{tabular}

Table 3.Descriptive statistics for age, gravidity, parity and abortion/miscarriage of 138 females.

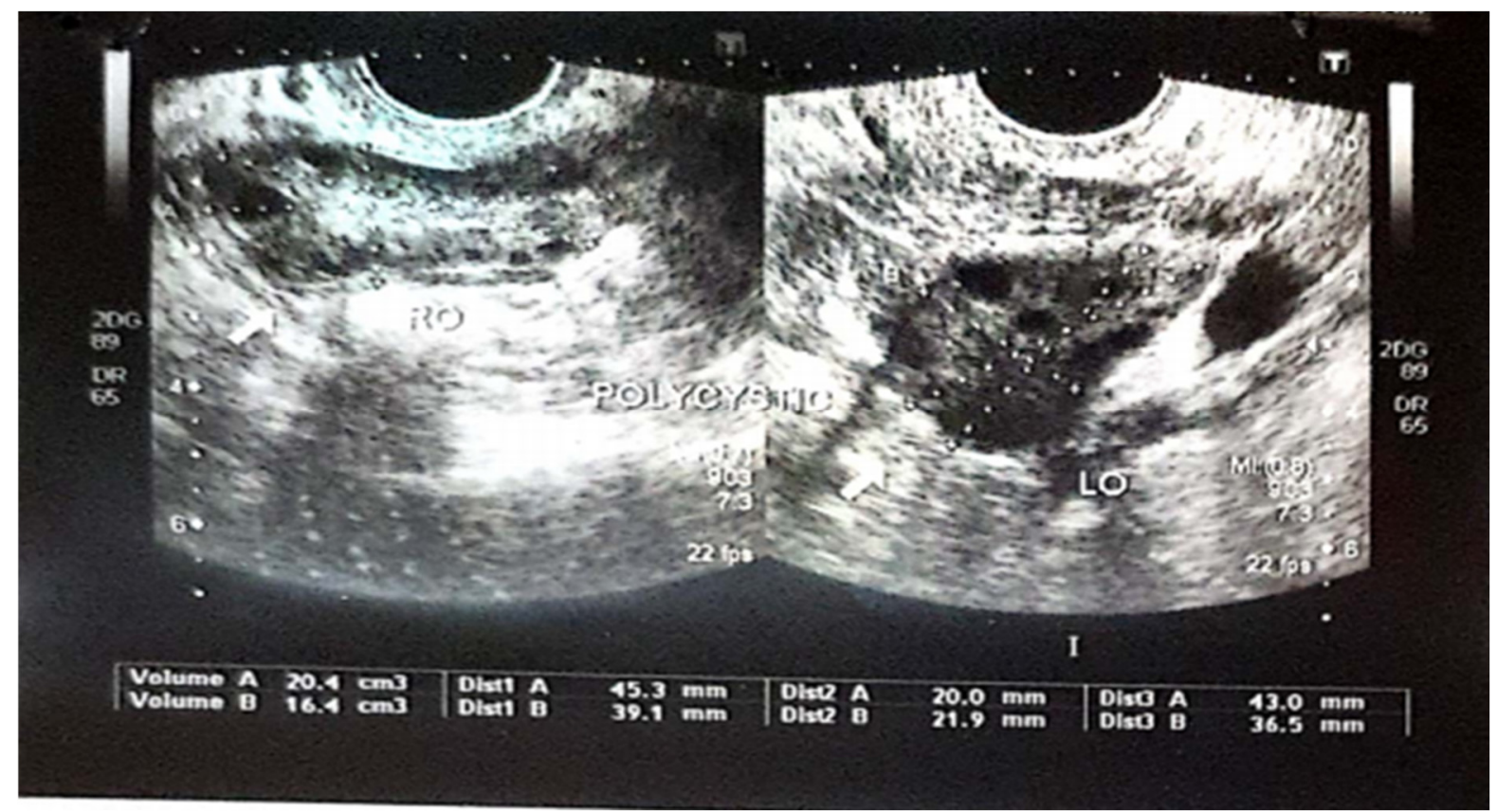

Polycystic ovaries showing multiple small follicles or string of pearls appearance and echogenic stroma with right ovarian volume of 20.4 grams and left ovarian volume of 16.4 grams.

PCOs was the most commonly seen pathology with highest frequency reported in $56(40.6 \%)$ patients. The second most commonly seen pathology was ovarian cyst $15(10.9 \%)$ followed by multiple fibroids $7(5.1 \%)$ as the third most commonly seen pathology.

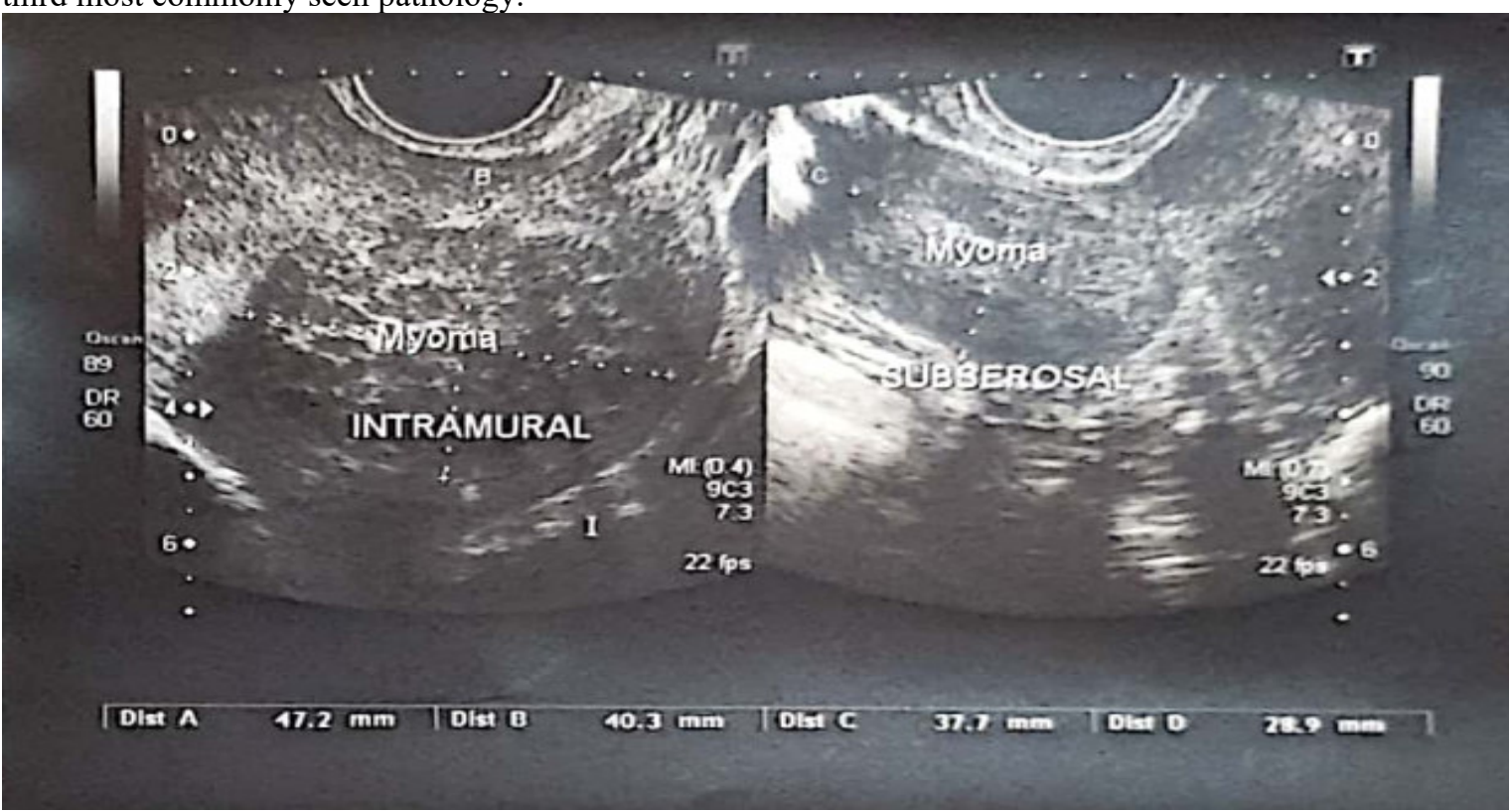

Intramural and subserosal fibroid measuring $47.2 \mathrm{~mm}$ x $40.3 \mathrm{~mm}$ and $37.7 \mathrm{~mm}$ x $28.9 \mathrm{~mm}$ respectively

Others were adenomyosis $1(0.7 \%)$,chronic cervicitis $1(0.7 \%)$, complex cyst $1(0.7 \%)$, dermoid cyst $1(0.7 \%)$, endometrial osseous metaplasia $1(0.7 \%)$, endometrial polyp $3(2.2 \%)$, endometrioma $5(3.6 \%)$, hemorrhagic cyst 4 (2.9\%), hydrosalpinx 3 (2.2\%), intramural fibroid 5 (3.6\%), nabothian cyst 3 (2.2\%), PID 4 (2.9\%), septated cyst 
$1(0.7 \%)$, small ovaries $2(1.4 \%)$, submucosal fibroid $1(0.7 \%)$, subserosal fibroid $6(4.3 \%)$, thickened endometrium $1(0.7 \%)$, while $17(12.3 \%)$ were not reported with any pathology and were considered to be normal.

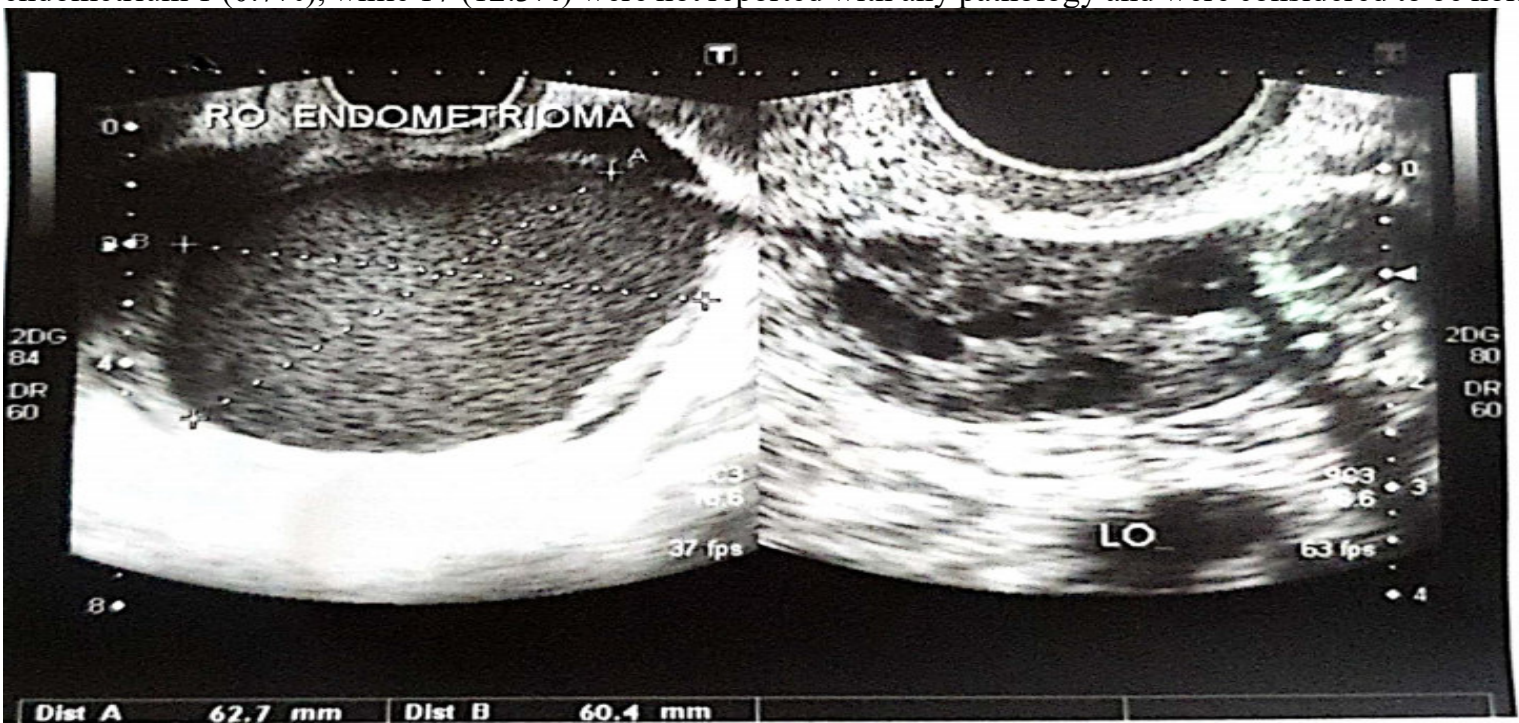

Right ovarian endometrioma measuring $62.7 \mathrm{~mm}$ x $60.4 \mathrm{~mm}$ and left polycystic ovary

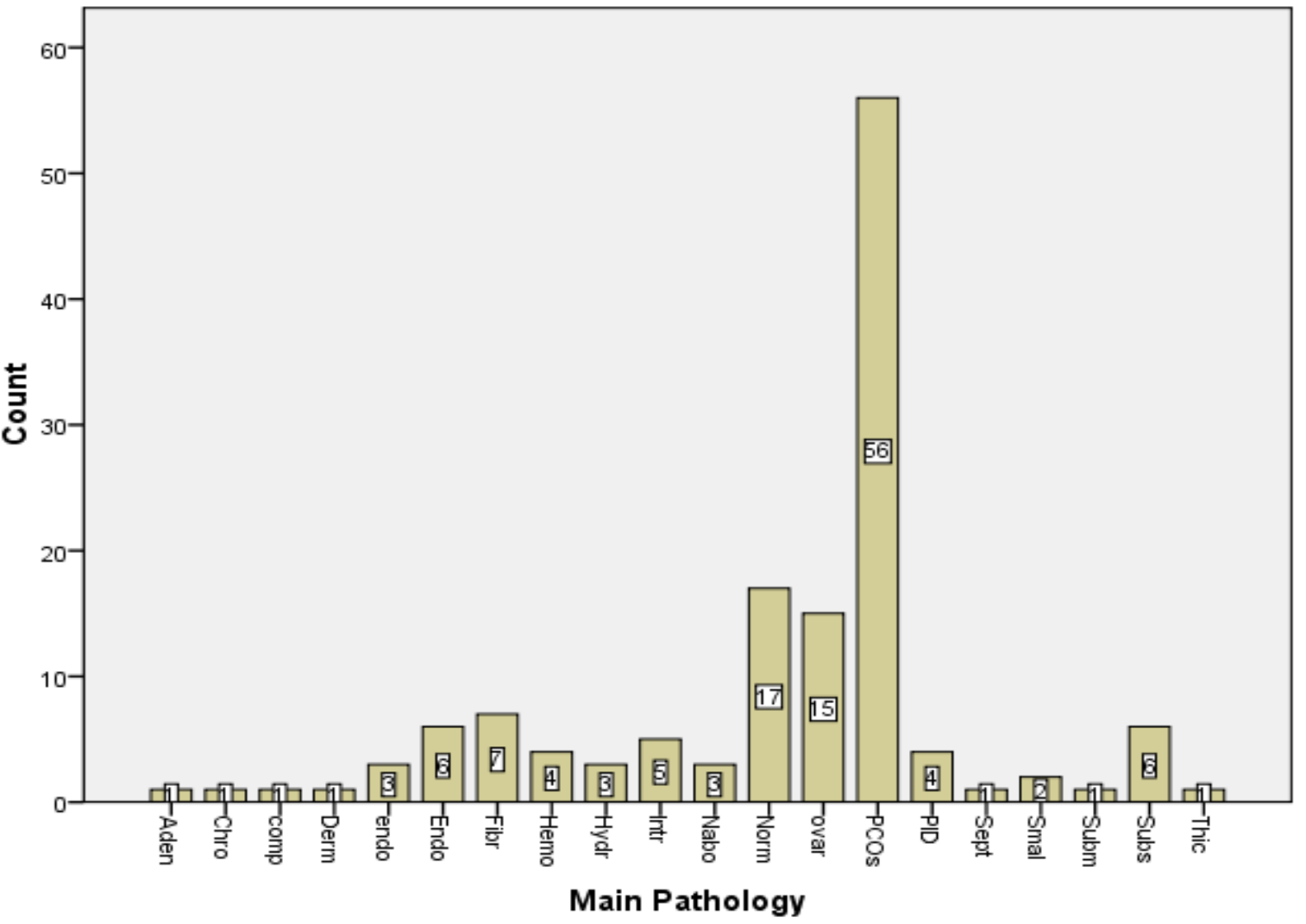

\section{Graph 1. Frequency distribution of all detected pathologies}

As for the location of pathology $1(0.7 \%)$ was reported in the anterior wall of uterus, $59(42.8 \%)$ pathologies in bilateral ovaries, $14(10.1 \%)$ in the left ovary, $3(2.2 \%)$ in the posterior wall of uterus and $12(8.7 \%)$ in the right ovary.

\section{Discussion}

The results of our study propose that there is higher occurrence of primary infertility in comparison to secondary infertility. These findings are similar to the findings of another study in which the prevalence of primary infertility was $69.5 \%$ and that of secondary infertility was $30.5 \%{ }^{17}$. The most common endocrine disorder in women is PCOS resulting in $75 \%$ cases of infertility due to absence of ovulation ${ }^{18}$. The amalgamation of anovulation and higher 
levels of androgens leads to polycystic ovaries. PCOS is correlated with other medical conditions in the female patients such as hypertension, obesity, insulin resistance, cardiovascular disease, diabetes mellitus type 2 , metabolic disorders, dyslipidemia, endometrial hyperplasia and carcinoma. Skin related symptoms of high androgen levels include alopecia, hirsutism, seborrhea, acne and virilization in critical cases. Infertility caused due to PCOS is related with higher levels of LH secretion and higher LH/FSH ratio ${ }^{19}$. According to our study the leading cause of infertility is also PCOS effecting almost $40.6 \%$ of cases in both primary and secondary infertile females.Genitourinary tract infections results in infertility in almost $15 \%$ of cases. At the age of 35, salpingitis causes subfertility in almost $2.5 \%$ of women. Pelvic Inflammatory disease (PID) occurs due to several infection causing agents such as Chlamydia Trachomatis, gonorrhea and multiple anaerobic infections of upper genital tract. Lower genital tract infection results from different bacterial, fungal and protozoan causes such as Bacterial Vaginosis, Candida Albicans and Trichomonasvaginalis. PID advances through vagina to cervix, uterus, endometrium and fallopian tubes ${ }^{20}$. In our study PID is the second main pathology diagnosed in the patients of primary as well as secondary infertility and were seen in $8.7 \%$ of cases. Uterine myomas are one of the remarkable cause of infertility and other medical conditions such as spontaneous abortion and complications in pregnancy. Submucosal fibroids effects fertility and upsurges the rate of spontaneous abortion and can be treated through myomectomy. Intramural fibroids also reduce fertility and surge the risk of spontaneous abortion but myomectomy is not the sole treatment to reinstate the normal position. However, subserosal fibroids do not effect fertility but they do upturn the risk of abortion. Complications associated to pregnancy caused by fibroids are intrauterine growth retardation, placental abruption, cesarean delivery, placenta previa ,retained placenta, postpartum hemorrhage, malpresentation and preterm labor. Fibroids may be symptomatic or asymptomatic, common symptoms include acute and chronic pelvic pain, urinary frequency, abnormal uterine bleeding and constipation. Almost $2.4 \%$ of women experience infertility due to fibroids ${ }^{21}$. In our study multiple fibroids were seen in $5.1 \%$ of females presenting with infertility and this was the third most common pathology seen in our study.

\section{Conclusion}

PCOS was the utmost collective pathology related with main and secondary infertility. The second and third most common pathologies were ovarian cyst and multiple fibroids respectively.

\section{References}

${ }^{1}$ Ogawa M, Takamatsu K, Horiguchi F. Evaluation of factors associated with the anxiety and depression of female infertility patients. BioPsychoSocial medicine. 2011 Dec;5(1):15.

${ }^{2} \mathrm{Naz}$ T, Hassan L, Gulmeen NF, Sultan S. Laparoscopic evaluation in infertility. J Coll Physicians Surg Pak. 2009 Nov;19(11):704-

${ }^{3}$ Unuane D, Tournaye H, Velkeniers B, Poppe K. Endocrine disorders \& female infertility. Best Practice \& Research Clinical Endocrinology \& Metabolism. 2011 Dec 1;25(6):861-73.

${ }^{4}$ Sami N, Saeed Ali T. Perceptions and experiences of women in Karachi, Pakistan regarding secondary infertility: results from a community-based qualitative study. Obstetrics and gynecology international. 2012;2012.

${ }^{5}$ Lindsay TJ, Vitrikas KR. Evaluation and treatment of infertility. Am Fam Physician. 2015 Mar 1;91(5):308-14.

${ }^{6}$ Shah HU, Sannananja B, Baheti AD, Udare AS, BadhePV.Hysterosalpingography and ultrasonography findings of female genital tuberculosis.Diagnostic and Interventional Radiology. 2015 Jan;21(1):10.

${ }^{7}$ Thangappah RB, Paramasivan CN, Narayanan S. Evaluating PCR, culture \& histopathology in the diagnosis of female genital tuberculosis. The Indian journal of medical research. 2011 Jul;134(1):40.

${ }^{8}$ Ramos RR, Gutiérrez GR, Monroy IA, Sánchez HG. Risk factors associated to female infertility. Ginecologia y obstetricia de Mexico. 2008;76(12):717-21.

${ }^{9}$ Pellati D, Mylonakis I, Bertoloni G, Fiore C, Andrisani A, Ambrosini G, Armanini D. Genital tract infections and infertility. European Journal of Obstetrics \& Gynecology and Reproductive Biology. 2008 Sep 1;140(1):3-11.

10 Weiss RV, Clapauch R. Female infertility of endocrine origin. ArquivosBrasileiros de Endocrinologia\&Metabologia. 2014 Mar;58(2):144-52.

${ }^{11}$ Sheethal C. Efficacy of TVS colourdoppler in comparison of primary infertility with endometrial biopsy.

${ }^{12}$ Imaoka I, Wada A, Matsuo M, Yoshida M, Kitagaki H, Sugimura K. MR imaging of disorders associated with female infertility: use in diagnosis, treatment, and management. Radiographics. 2003 Nov;23(6):1401-21.

${ }^{13}$ MALIK SA, MALIK S, MAQBOOL A. Comparison of Transabdominal and TransvaginalSonography in the Diagnosis of Ectopic Pregnancy.2010 Jan 4(1):22-27.11w

${ }^{14}$ Widrich T. Role of Ultrasonography in Infertility.InOffice-Based Infertility Practice 2002 (pp. 39-48). Springer, New York, NY.

${ }^{15}$ Fraser MA, Agarwal S, Chen I, Singh SS. Routine vs. expert-guided transvaginal ultrasound in the diagnosis of endometriosis: a retrospective review. Abdominal imaging. 2015 Mar 1;40(3):587-94.

${ }^{16}$ Brassard M, AinMelk Y, Baillargeon JP.Basic infertility including polycystic ovary syndrome.Medical Clinics 
of North America. 2008 Sep 1;92(5):1163-92.

${ }^{17}$ Masoumi SZ, Parsa P, Darvish N, Mokhtari S, Yavangi M, Roshanaei G. An epidemiologic survey on the causes of infertility in patients referred to infertility center in Fatemieh Hospital in Hamadan. Iranian journal of reproductive medicine. 2015 Aug;13(8):513.

${ }^{18}$ Messinis IE, Messini CI, Dafopoulos K. Obesity in PCOS and Infertility. Obesity: A ticking time bomb for reproductive health. 2013:99-116

${ }^{19}$ Barbosa G, de Sá LB, Rocha DR, Arbex AK. Polycystic ovary syndrome (PCOS) and fertility. Open Journal of Endocrine and Metabolic Diseases. 2016 Jan 12;6(01):58.

${ }^{20}$ Chhetri A, Biswas SC, Gupta K. Infection and Infertility.Practical Guide in Infertility. 2018 Apr 30:107.

${ }^{21}$ Olive DL, Pritts EA. Fibroids and reproduction. InSeminars in reproductive medicine 2010 May (Vol. 28, No. 03, pp. 218-227).C Thieme Medical Publishers. 